\title{
The pattern of prostate cancer local recurrence after radiation and salvage cryoablation
}

\author{
Chee Kwan Ng, MD; Naji J.Touma, MD, FRCSC; Venu Chalasani, MD; Madeleine Moussa, MD, FRCPC; \\ Donal B. Downey, MD, FRCPC; Joseph L. Chin, MD, FRCSC
}

Cite as: Can Urol Assoc J 2011;5(6):E125-E128; http://dx.doi.org/10.5489/cuaj.09116. Epub 2011 Jan 13.

\section{Abstract}

Objective: We assessed the pattern of local recurrence after salvage cryoablation of the prostate, and the impact of local recurrence on intermediate-term outcome.

Methods: One hundred twenty-two patients who underwent salvage cryoablation were studied after a mean follow-up of 56 months. Serial prostate biopsy was carried out after cryoablation. The histopathology of prostate biopsies before and after cryoablation were compared. The prognostic value of post-cryoablation biopsy was assessed with the Cox regression method.

Results: $23.1 \%$ of patients had a positive biopsy for prostate cancer following salvage cryoablation. Most cancer recurrences occurred in the apex $(51.5 \%)$, base $(21.2 \%)$ and seminal vesicles $(18.2 \%)$. The presence of cancer at the base of the prostate was found to be a prognostic factor for eventual biochemical failure. Overall 5-year biochemical disease-free survival (bDFS) was 28\%, however patients with cancer at the base of the prostate had a 5-year bDFS of $0 \%$.

Conclusion: Cancer recurrences occurred in areas where aggressive freezing was avoided as it might result in serious problems (e.g., urethro-rectal fistula and incontinence). Post-cryoablation biopsies and the location of persistent disease are of prognostic value.

\section{Introduction}

Recent data from the Cancer of the Prostate Strategic Urologic Research Endeavor (CAPSURE) show that prostate cancer recurs in up to $63 \%$ of patients after external beam radiotherapy. ${ }^{1}$ The optimal management of patients with prostate cancer recurring locally after radiotherapy is not yet determined. The eradication of local recurrence can be attempted by salvage prostatectomy, brachytherapy or cryoablation. The alternative is systemic treatment with hormonal therapy, which is not curative; however this is the most common salvage treatment, used in about $93 \%$ of patients. ${ }^{1}$ Treatment decisions are often based on patient comorbidities and physician expertise and preference. Patients who have failed radiotherapy and have limited life expectancy or have multiple comorbidities would be precluded from salvage surgery. At our centre, salvage cryoablation of the prostate has been offered as a minimally invasive treatment option for this cohort for the past 15 years. We have reported on the short-term biochemical results and acceptable morbidity previously. ${ }^{2}$ Our cohort of patients has had a mean follow-up of 56 months, and intermediate-term results have now been analyzed. ${ }^{3}$ As part of our research protocol, these patients have had serial transrectal ultrasound guided biopsies postcryoablation. The aim of this study was to look at the pattern of local recurrence in the prostate, as well as the impact of local recurrence on intermediate-term outcome.

\section{Materials and methods}

Between March 1995 and December 2001, we performed salvage cryoablation on 122 patients with biochemical failure after radiotherapy (external beam radiotherapy in 121 patients, brachytherapy in 1 patient). All but 2 of the patients had sextant biopsy-proven local recurrence, and all patients had negative computerized tomography (CT) scans and radionuclide bone scans. The median age was 70 years (range 53.6-81.7). Median prostate-specific antigen (PSA) before radiotherapy was $12.2 \mathrm{ng} / \mathrm{mL}$ (range 3.3-100). Clinical stage distribution was 15\% T1, 70\% T2, 14\% T3, and $1 \%$ T4. Pathologic grading of initial disease was $68 \%$ Gleason $\leq 6,32 \%$ Gleason $\geq 7$. Median PSA at the time of cryoablation was $6.2 \mathrm{ng} / \mathrm{mL}$ (range 0-36.4). Pathologic grading of local recurrence was $22 \%$ Gleason $\leq 6,78 \%$ Gleason $\geq 7$. Seventy-one percent had 3 to 6 months of neoadjuvant hormonal therapy for downsizing. The Cryocare (Endocare, Inc., Irvine, CA) system was used in all cases, except for the initial 11 in which a Candela (Candela, Inc. Boston, MA) system was used. Transrectal ultrasound with 3-D imaging was used for placement of cryoprobes, as previously reported. Proper probe placement was confirmed on 3-D ultrasound in terms of inter-probe distances, probe orientation and depth. ${ }^{4,5}$ In most cases, 5 cryoprobes (range 2-8) were inserted with a transperineal approach using the 
Seldinger technique after initial prostatic access by needle puncture. All patients underwent 2 freeze-thaw cycles, with the urethra protected by a urethral warmer (Cook Medical, Inc., Bloomington, IN). Monitoring of the freezing process was achieved with real-time ultrasound visualization of the iceball, as well as temperature monitoring by way of 3 thermocouples inserted into the periprostatic area (left and right neurovascular bundle, midline near apex). The bladder was drained postoperatively with a urethral Foley catheter for 1 day, and a suprapubic cystostomy catheter for 3 weeks. Serial serum PSA levels were done at 3, 6, 12, 24 and 36 months, postoperatively. Biochemical failure was defined as the serum PSA reaching $2 \mathrm{ng} / \mathrm{mL}$ above the nadir (Phoenix definition). Transrectal ultrasound guided biopsy was performed at 6, 12 and 24 months whenever logistically feasible. After 24 months, biopsy was performed when clinically indicated. Biopsies were obtained from 4 quadrants of the prostate, as well as from sonographically and/ or clinically suspicious areas initially; subsequently, sextant biopsies were performed. Mean follow-up was 56 months (range 9-111 months).

Statistical analysis was performed with the SPSS 11.0 program (Lead Technologies, Inc, Charlotte, NC). Differences in proportions were assessed with the chi-square test. Survival analysis was performed with the Kaplan-Meier method, and univariable analysis was performed with the Cox regression model. This study was conducted in accordance with the Declaration of Helsinki.

\section{Results}

One hundred seventeen of 122 patients underwent repeated systematic biopsy after cryoablation. The remaining 5 patients were followed up in their home provinces. One-, 2 - and 5-year freedom from local recurrence was 95\%, 90\% and $73 \%$, respectively. Overall, 27 patients $(23.1 \%)$ had biopsies positive for prostate cancer, with a median of 1 core positive (range 1-3). Gleason score distribution was $27.3 \%$ Gleason $\leq 6,72.7 \%$ Gleason $\geq 7$. The location of cancers after cryoablation is shown in Table 1. The location of cancers before and after cryoablation is compared in Table 2. Before cryoablation, cancer was quite evenly distributed throughout the base (36.7\%), mid (26.6\%) and apex (33.3\%) of the prostate. In contrast, most of the cancer recurrences after cryoablation were at the apex $(51.5 \%)$, base $(21.2 \%)$ and seminal vesicles (18.2\%). The presence of cancer at the base was predictive of biochemical failure, with a hazard ratio of $0.376(p=0.014)$ (Table 3$)$. The overall presence of cancer on biopsy, Gleason score and the presence of cancer at the mid, apex and seminal vesicles did not correlate with biochemical failure. Overall mean biochemical recurrence-free survival was 43 months, and the 5-year biochemical recurrence-free survival was $28 \%$. The small

\begin{tabular}{lc} 
Table 1. Distribution of cancer in post-cryoablation biopsies \\
\hline Location & Number \\
\hline Base only & 3 \\
Base + mid & 1 \\
Base + apex & 3 \\
Apex only & 11 \\
Apex + mid & 1 \\
Apex + seminal vesicles & 1 \\
Apex + TURP specimen & 1 \\
Mid only & 1 \\
Seminal vesicles & 5 \\
\hline Total & 27 \\
\hline TURP = transurethral resection of the prostate.
\end{tabular}

group of patients with positive biopsies at the base had a 5 -year survival of $0 \%$ and a mean biochemical recurrencefree survival of only 20 months. This difference in survival is demonstrated graphically by plotting the Kaplan-Meier curves (Fig. 1).

\section{Discussion}

Salvage cryoablation of the prostate is a locally curative option for patients with organ confined recurrence after radiotherapy; it is not intended to treat extra-prostatic disease. The long-term efficacy of salvage cryoablation has not been fully documented. The procedure is mainly performed in a limited number of centres in North America and survival data is scanty. Some centres have reported encouraging short term data, with biochemical recurrence-free survival up to

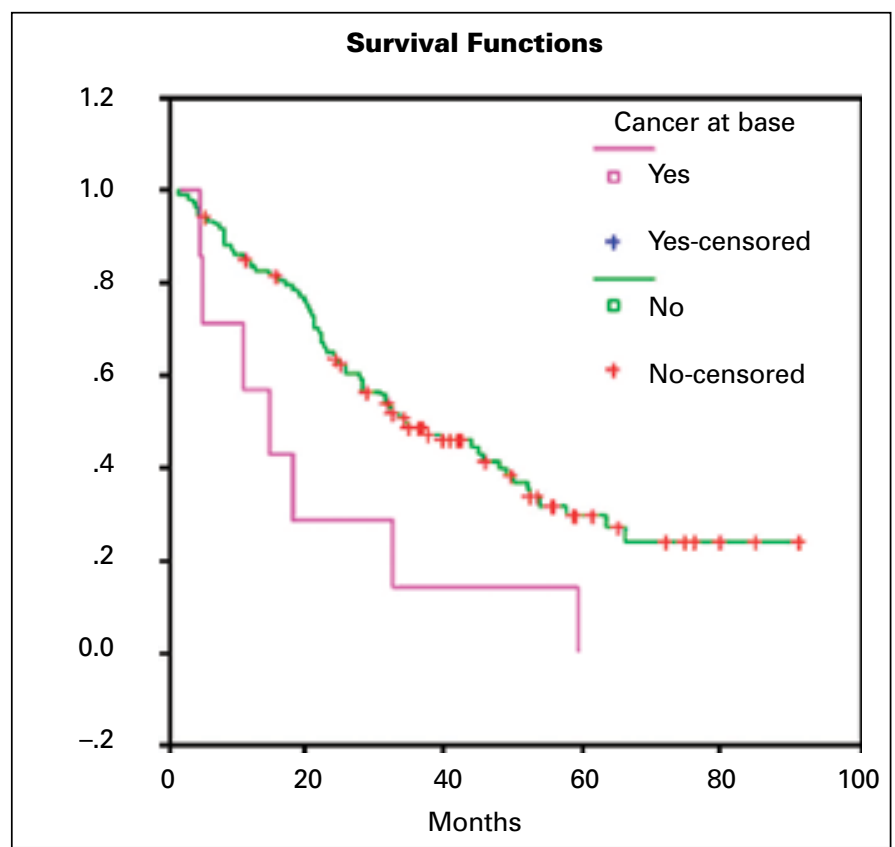

Fig. 1. Impact of positive biopsy at base on freedom from biochemical failure $(p=0.0109)$. 
Table 2. Location of cancer on biopsy

\begin{tabular}{|c|c|c|c|c|c|c|}
\hline & & \multicolumn{4}{|c|}{ Location of positive biopsies } & \multirow{2}{*}{ Total } \\
\hline & & Base & Mid & Apex & Seminal vesicle & \\
\hline \multirow{2}{*}{$\begin{array}{l}\text { No. of positive } \\
\text { biopsies }\end{array}$} & Pre-cryoablation & $76(36.7 \%)$ & $55(26.6 \%)$ & $69(33.3 \%)$ & $7(3.4 \%)$ & $207(100 \%)$ \\
\hline & Post-cryoablation & $7(21.2 \%)$ & $3(9.1 \%)$ & 17 (51.5\%) & $6(18.2 \%)$ & $33(100 \%)$ \\
\hline
\end{tabular}

$74 \%$ at 2 years. ${ }^{6}$ In the intermediate term, however, a positive biopsy rate of $21 \%,{ }^{7}$ and a 5 -year disease-free survival of $40 \%$ to $55 \%$ and 5 -year disease specific survival of $79 \%$ has been reported. ${ }^{8,9}$

Careful selection of patients is important for good outcomes. Patients with low pre-cryoablation PSA, Gleason score and clinical stage have superior disease-free survival. ${ }^{2,8}$ For the purpose of reporting outcomes, we have adopted the Phoenix definition of biochemical failure, ${ }^{10}$ as there is no standard definition for biochemical failure after salvage cryoablation, due to the lack of large data sets with salvage cryoablation. Our updated analysis confirms our previous findings that patients with low pre-cryoablation PSA have improved biochemical recurrence-free survival. ${ }^{2,3}$ For patients with pre-cryoablation PSA $<4 \mathrm{ng} / \mathrm{mL}$, the 5 -year biochemical recurrence-free survival was $56 \%$, compared with patients with PSA $>10 \mathrm{ng} / \mathrm{mL}$, where the 5 -year biochemical recurrence-free survival was only $14 \%{ }^{3}$

Because our patients underwent a protocol of regular biopsy after cryoablation, we have been able to evaluate the efficacy of our method of cryoablation by analyzing the pathological outcomes. Previously, we reported that $42 \%$ of patients had evidence of residual viable prostate glandular tissue on repeat biopsy. ${ }^{11}$ Similarly, Izawa and colleagues reported that complete ablation of the prostate gland and tumour was difficult to achieve with salvage cryoablation, however, the finding of atypical or normal epithelial tissue in biopsy specimens after salvage cryoablation was not predictive of biochemical failure. ${ }^{12}$ In this study, we focused on the pattern of cancer persistence or recurrence after salvage cryoablation. We found a positive biopsy rate of $23.1 \%$, and a distribution of recurrent cancer mostly at the apex, base and seminal vesicles. Similarly, in a series of patients who underwent primary cryoablation of the prostate, Shinohara and colleagues found that most of the recurrences occurred in the apex and seminal vesicles. ${ }^{13}$ In a small series of patients who underwent cryoablation followed by radical prostatectomy, Pisters and colleagues found persistent viable areas posterolateral in the prostatic base, which required modification of the probe position for subsequent patients. ${ }^{14}$ We postulate that a major factor for recurrent cancer is the relatively conservative freezing in areas of the prostate where vital structures need to be preserved. To avoid damage to the external urethral sphincter, the surgeon tends to limit the extent of the iceball near this structure at the apex and consequently the apex is a common site of recurrent cancer ( $51.5 \%$ in our series). To avoid urethro-rectal fistula formation, the surgeon usually stops the freezing process once the edge of the iceball reaches the muscularis propria of the rectum. Hence the tips of the seminal vesicles, which are in close proximity to the rectum and which may be outside the limit of the spherical iceballs, are often inadequately ablated, leading to recurrent cancer in that area. Likewise, the finding of recurrent cancer at the base may be explained by suboptimal tissue ablation at the edges of the iceball. Other reasons for failure include the presence of extra-prostatic disease which was not visible on the imaging modalities available between 1995 and 2001. The presence of positive biopsies at the base may also be indicative of seminal vesicle involvement.

The use of the urethral warmer protecting periurethral tissue may also contribute to persistent cancer, although this is not evident in our series. Others have been shown anecdotally that viable periurethral tissue is consistently found in prostatectomy specimens obtained after cryoablation with the urethral warmer in use..$^{14,15}$ In their small series of radical prostatectomy following cryoablation, Pisters and colleagues found that 2 out of 7 patients had viable periurethral cancer. ${ }^{14}$

Improvements in technology have made possible the development of smaller diameter cryoprobes which are inserted without the need for stab incisions or tract dilatation. The newer systems (e.g., SeedNet, Oncura, Arlington Heights, IL) theoretically allow for better contouring of the prostate, and perhaps a more even distribution of the freezing process. Long-term follow-up is pending. ${ }^{16}$ However, even with this system, it may still not be possible to achieve ablation of prostate tissue safely in areas near the rectum and the urethral sphincter. Interestingly, Cytron and colleagues have reported a novel method of active rectal wall protection using active thawing by 2 cryo-needles inserted in the space between the prostate and rectum. ${ }^{17}$ It is thought that

\begin{tabular}{lcc}
\hline $\begin{array}{l}\text { Table 3. Univariable analysis of prognostic factors for } \\
\text { biochemical failure }\end{array}$ & p value & HR (95\% Cl) \\
\hline Cancer at biopsy & 0.069 & $0.617(0.366-1.038)$ \\
\hline Gleason score & 0.553 & $0.708(0.226-2.213)$ \\
\hline Number of positive cores & 0.077 & $1.698(0.944-3.054)$ \\
\hline Cancer at base & 0.014 & $0.376(0.172-0.824)$ \\
\hline Cancer at mid & 0.065 & $0.329(0.101-1.072)$ \\
\hline Cancer at apex & 0.227 & $0.680(0.364-1.271)$ \\
\hline Cancer at seminal vesicle & 0.677 & $1.279(0.401-4.080)$ \\
\hline Cl: confidence interval. & &
\end{tabular}


this allows low temperatures to be achieved at the peripheral zone of the prostate without inducing inadvertent injury to the rectal wall. The long-term effectiveness of this method remains to be demonstrated. The technique of hydrodissection has been described in the context of interstitial microwave thermal therapy for prostate cancer. ${ }^{18}$ In this method, an injection of normal saline into the space between the rectum and the prostate causes the rectum to be separated further from the prostate; this allows cytotoxic temperatures to be delivered to the posterior prostate without causing damage to the rectum. It is possible that this technique could be used for cryoablation of the prostate as well. Nevertheless, ongoing improvements in cryoablation technology and ultrasound monitoring techniques will continue to lower the likelihood of inadvertent injury to adjacent structures.

In our study, the presence of cancer at the base of the prostate was found to be a statistically significant prognostic factor for biochemical recurrence-free survival $(p=0.014)$. All patients, albeit only a small group, with cancer at the base of the prostate eventually developed biochemical recurrence. This finding appears analogous to the setting after radical prostatectomy, where positive margins at the prostatic base or bladder neck are a significant prognostic factor for recurrence. ${ }^{19-20}$ This is perhaps a reflection of more extensive disease, such as seminal vesicle involvement; 4 out of 7 patients with cancer at the base had cancer at other locations as well (Table 1). Improving pre-treatment staging techniques, such as targeted biopsies of the seminal vesicles prior to consideration of local salvage, may avoid this in the future. This finding has implications on post-cryoablation biopsy technique. The method of biopsy in our study was either quadrant or sextant, which raises the possibility of sampling error, especially when compared with more recent cryoablation series in which more extensive biopsies are performed. However, as patients underwent serial biopsy, the cumulative number of biopsies performed would have minimized sampling errors. Biopsy in this setting is often more difficult as the prostate is shrunken and fibrotic, and the risk of complications, such as fistula formation, is higher. Limiting the number of cores biopsied and concentrating on the base, which has the best prognostic yield, would be prudent; nevertheless, we acknowledge that the number of biopsies performed is one of the limitations of our study.

\section{Conclusion}

Cancer recurrences occurred in areas where aggressive freezing was avoided as it might result in serious problems (e.g., incontinence and recto-urethral fistula). Postcryoablation biopsies should include samples from the base of the prostate for their prognostic value. Further refinement in candidate selection and in cryoablation techniques would enhance the utility of salvage cryoablation following radiation for prostate cancer.
From the Division of Urology, Department of Pathology and Department of Radiology, London Health Sciences Centre, University of Western Ontario, London, ON

Competing interests: None declared.

This paper has been peer-reviewed.

\section{References}

1. Agarwal PK, Sadetsky N, Konety BR, et al. Cancer of the Prostate Strategic Urological Research Endeavor (CaPSURE): Treatment failure after primary and salvage therapy for prostate cancer: likelihood, patterns of care, and outcomes. Cancer 2008;112:307-14.

2. Chin JL, Pautler SE, Mouraviev V, et al. Results of salvage cryoablation of the prostate after radiation: identifying predictors of treatment failure and complications. J Urol 2001;165:1937-41; discussion 1941-2.

3. $\mathrm{Ng} \mathrm{CK}$, Moussa M, Downey DB, et al. Salvage cryoablation of the prostate: followup and analysis of predictive factors for outcome. J Urol 2007;178:1253-7; discussion 1257.

4. Chin JL, Downey DB, Onik G, et al. Three-dimensional prostate ultrasound and its application to cryosurgery. Tech Urol 1996;2:187-93.

5. Chin JL, Downey DB, Mulligan $M$, et al. Three-dimensional transrectal ultrasound guided cryoablation for localized prostate cancer in nonsurgical candidates: a feasibility study and report of early results. J Urol 1998;159:910-4.

6. Ghafar MA, Johnson CW, De La Taille A, et al. Salvage cryotherapy using an argon based system for locally recurrent prostate cancer after radiation therapy: the Columbia experience. J Urol 2001;166:1333-7; discussion 1337-8.

7. Izawa JI, Perrotte P, Greene GF, et al. Local tumor control with salvage cryotherapy for locally recurrent prostate cancer after external beam radiotherapy. J Urol 2001;165:867-70.

8. Izawa JI, Madsen LT, Scott SM, et al. Salvage cryotherapy for recurrent prostate cancer after radiotherapy: variables affecting patient outcome. J Clin Oncol 2002;20:2664-71.

9. Pisters LL, Rewcastle JC, Donnelly BJ, et al. Salvage prostate cryoablation: initial results from the cryo on-line data registry. J Urol 2008; 180:559-63; discussion 563-4.

10. Pickles T, Kim-Sing C, Morris WJ, et al. Evaluation of the Houston biochemical relapse definition in men treated with prolonged neoadiuvant and adjuvant androgen ablation and assessment of follow-up lead-time bias. Int J Radiat Oncol Biol Phys 2003;57:11-8.

11. Chin JL, Touma N, Pautler SE, et al. Serial histopathology results of salvage cryoablation for prostate cancer after radiation failure. J Urol 2003;170:1199-202.

12. Izawa Jl, Morganstern N, Chan DM, et al. Incomplete glandular ablation after salvage cryotherapy for recurrent prostate cancer after radiotherapy. Int J Radiat Oncol Biol Phys 2003;56:468-72.

13. Shinohara K, Rhee B, Presti JC Jr, et al. Cryosurgical ablation of prostate cancer: patterns of cancer recurrence. J Urol 1997;158:2206-9; discussion 2209-10.

14. Pisters LL, Dinney CP, Pettaway CA, et al. A feasibility study of cryotherapy followed by radical prostatectomy for locally advanced prostate cancer. J Urol 1999;161:509-14.

15. Donnelly BJ, Saliken JC, Ali-Ridha N, et al. Histological findings in the prostate two years following cryosurgical ablation. Can J Urol 2001;8:1237-9.

16. Han KR, Cohen JK, Miller RJ, et al. Treatment of organ confined prostate cancer with third generation cryosurgery: preliminary multicenter experience. J Urol 2003;170:1126-30.

17. Cytron $S$, Paz A, Kracchick $S$, et al. Active rectal wall protection using direct transperineal cryo-needles for histologically proven prostate adenocarcinomas. Eur Urol 2003;44:315-20; discussion 320-1.

18. Sherar MD, Gertner MR, Yue CK, et al. Intersititial microwave thermal therapy for prostate cancer: method of treatment and results of a phase I/II trial. J Urol 2001;166:1707-14.

19. Blute ML, Bostwick DG, Bergstralh EJ, et al. Anatomic site-specific positive margins in organ-confined prostate cancer and its impact on outcome after radical prostatectomy. Urology 1997;50:733-9.

20. Aydin H, Tsuzuki T, Hernandez D, et al. Positive proximal (bladder neck) margin at radical prostatectomy confers greater risk of biochemical progression. Urology 2004;64:551-5.

Correspondence: Dr. Joseph L. Chin, Victoria Hospital 800 Commissioners Rd East, London, ON N6A 4G5; joseph.chin@hths.on.ca 